\title{
Taste Pore
}

National Cancer Institute

\section{Source}

National Cancer Institute. Taste Pore. NCI Thesaurus. Code C33738.

The opening at the apex of the taste bud. 